\title{
Seeing is believing: Wnt3 localization in the gut epithelium
}

\author{
Cell Research (2016) 26:515-516. doi:10.1038/cr.2016.41; published online 25 March 2016
}

\begin{abstract}
Wnt morphogens are notoriously elusive proteins. Thanks to a recent study published in Nature, Clevers and colleagues give us a first glimpse of a mammalian Wnt in action in the gut epithelium.
\end{abstract}

Tissue function is crucially dependent on the proper spatial organization of cells in three dimensions. Cellular architecture is established in part by morphogens or signaling molecules expressed in concentration gradients along the forming tissues. In Lewis Wolpert's famous French flag model, distinct biological effects are induced depending on the position of cells within the morphogen gradient. Several types of morphogens have been described including transcription factors in the Drosophila syncytial embryo, metabolites such as retinoic acid and of course secreted growth factors such as Wnt, TGF $\beta$ and Shh.

The importance of morphogens in regulating pattern formation during embryonic development and tissue regeneration is well described, but what is not so clear is how gradients of morphogen activity are established across tissues. In a recent issue of Nature, Clevers and colleagues provide new insight into this question by visualizing the distribution of one particular morphogen, called Wnt3, within the intestinal crypt epithelium [1]. The gut epithelium is a self-renewing tissue composed of highly repetitive units called crypts and villi. The former are formed by invagination of the epithelium and are largely made up of intensely proliferating intestinal stem cells (ISCs) and progenitor populations. As part of their normal life cycle, most epithelial cells are forced out of the crypt and terminally differentiate into the specialized cells of the villus compartment. Proliferation in the crypts has long been assumed to be governed by a gradient of Wnt activity with the ISCs at the crypt bottom exposed to maximal levels of Wnt signaling (Figure 1A). Consistent with this notion, the expression levels of Wnt target genes is typically high in cells occupying the crypt bottom and declines as cells move out of the stem cell compartment higher up the crypt. Moreover, recent evidence has shown that the high Wnt activity of ISCs depends on their close contact with neighboring post-mitotic Paneth cells. Paneth cells not only play important antimicrobial functions, but also orchestrate crypt growth by releasing Wnts (e.g., Wnt3 and Wnt11) and other promitotic factors from the crypt base [2]. The question posed by Farin et al. was whether Paneth cell-derived Wnts act only locally on adjacent ISCs or whether long-range mechanisms exist to propel Wnts beyond the stem cell zone.

To track Wnt proteins, Farin et al. devised a strategy that bypassed the difficult task of generating reliable Wnt antibodies and instead created a HA epitope-tagged Wnt3 knockin mouse (Wnt3 ${ }^{\mathrm{HA} / \mathrm{HA}}$ ). Since HA-Wnt3 was functionally active both in vivo and in organoid cultures, the authors could then use these mice to visualize HA-Wnt 3 in intestinal crypts by simply performing anti-HA antibody stainings in combination with signal amplification steps. By staining both permeabilized and non-permeabilized tissues, the authors concluded that HA-Wnt3 was primarily at the external surfaces of basolateral membranes of crypt cell and conspicuously absent from the secretory granules in Paneth cells. With this elegant genetic tool, Farin et al. then examined how HA-Wnt3 is transferred between producing and receiving cells. Initial experiments showed that wildtype organoids could not rescue growth of Wnt 3 mutant organoids in co-culture assays and addition of neutralizing antibodies did not block growth of Wnt $3^{\mathrm{HA}}$

HA organoids. These results suggested that active Wnt3 does not function as a diffusible factor. To examine how far membrane-bound HA-Wnt3 traveled from its source, Farin et al. then employed a cell re-association assay. Non-labeled HA-Wnt3-producing cells were combined with wild-type dsRED cells and allowed to develop into large mosaic organoids. At the interfaces between producing and receiving cells, HA-Wnt3 penetrated only one or two cell diameters into HA-Wnt3-negative domains. These data thus suggest that Wnt 3 transfer is limited in range and involves direct contact between Wnt3producing Paneth cells and ISCs.

Wnts trigger their biological effects by binding to Frizzled receptors. Downstream signaling is terminated at the cell surface by induction of transmembrane E3 ubiquitin ligases, Rnf43 and Znrf3, which target Frizzleds to lysosomal degradation [3]. The negative action of Rn43/Znrf3 on Frizzled activity is inhibited by the association of Lgr $4 / 5$ proteins with their secreted ligands Rspondins. This complex recruits Rnf43/ Znrf3 away from Frizzleds and results in perdurance of Wnt stimulation. Armed with this knowledge, Farin et al. next manipulated Frizzled cell surface levels indirectly by modulating $\mathrm{R}$-spondin 1 


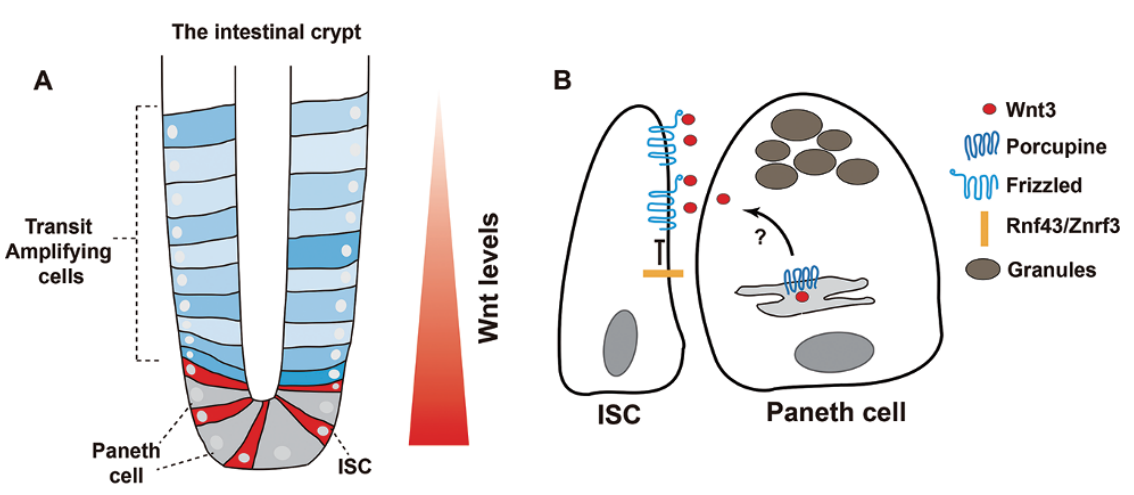

Figure 1 Schematic view of the intestinal crypt and Wnt transfer from Paneth cells to intestinal stem cells (ISCs). (A) A gradient of Wnt activity subdivides the crypt into a stem cell zone composed of ISCs and Paneth cells at the crypt bottom and a transit-amplifying zone made up of progenitor cells. (B) Wnt secretion in Paneth cells depends partly on Porcupine and once released Wnts are captured by Frizzled receptors on ISCs. Frizzled turnover is mediated by Rnf43/Znrf3. The molecular mechanisms underlying Wnt secretion and transfer from Paneth cells to ISCs remain unclear.

and Rnf43 expression in organoids. These experiments showed that Frizzleds are required to anchor HA-Wnt3 at the cell surface. Wnt ligands are lipid modified, which is required for its biological activity, but also presents somewhat of a conundrum for understanding how it acts at a distance [4]. Lipidation is mediated by, Porcupine, an ER-resident protein and o-aceyl transferase that palmitoylates Wnts. The authors therefore explored HA-Wnt3 processing in Paneth cells using an inhibitor of Porcupine. Indeed, treatment of organoids with Porcupine inhibitor led to a loss of HA-Wnt3 at cell membranes and corresponding cytoplasmic accumulation in Paneth cells within 24 $h$, consistent with Porcupine's essential role in secretion and lipid modification of Wnts.

Evidence so far suggested that HAWnt3 is secreted by Paneth cells via Porcupine and captured by Frizzleds in surrounding ISCs (Figure 1B). Because ISCs are continuously undergoing cell division, Farin et al. then asked whether further propagation of HAWnt 3 beyond the stem cell compartment depends on ISC cell division. Pulsechase experiments were conducted by washing out organoids treated with
Porcupine inhibitor to release newly produced HA-Wnt3. After 48 h, crypts regained a normal gradient of HA-Wnt3 expression. However, in the presence of mitotic inhibitors blocking Egfr, Mek or Cdk4/6, HA-Wnt3 remained localized near producing cells. Therefore these data suggested that Wnt 3 once bound to ISCs travels up the crypt by piggybacking on proliferating ISCs and their progeny rather than diffusion.

Now that we have a clear view of at least one mammalian Wnt in its native environment, the next question becomes one of mechanism. More specifically, how is Wnt3 transferred from producing cell to receiving cell. Here numerous options exist. Following post-translational modifications in the ER and Golgi, Wnts are known to associate with the sorting receptor, Wls, and are released into the extracellular space [5]. Endosomes and the retromer complex recycle Wls, allowing for multiple rounds of Wnt secretion. Once released, Wnts can utilize several modes of transport including lipoprotein particles, exosomes and cytonemes [6-8]. To complicate matters further, spreading of Wnts also depends on interactions with various heparan sulfate proteoglycans that reside in membranes and in the extracellular matrix. How this relates to Wnt3 transport in the crypt epithelium or other tissue types is unknown. The only available clue is that retromer transport appears not to be necessary for Wnt signaling in the gut [9]. Hopefully, purification of HA-Wnt3 from the gut epithelium combined with the use of mass spectrometry can shed light on these issues.

Finally, given that other sources of Wnts can sustain crypt homeostasis in vivo in the absence of Paneth cells [10], it will also be interesting to see how these Wnts get to their target cells. For example, an important source of Wnts, absent from the organoid system are surrounding stromal cells. How do these stromally derived Wnts traverse the basement membrane and which epithelial cells do they target? Obviously, these fundamental questions will continue to challenge researchers for several years. However, having now found Wnt3, we are no longer in the dark.

\section{Alex Gregorieff ${ }^{1}$, Jeffrey L Wrana ${ }^{1,2}$}

${ }^{1}$ Lunenfeld-Tanenbaum Research Institute, Mount Sinai Hospital, Sinai Health System, 600 University Avenue, Toronto, Ontario M5G 1X5, Canada; ${ }^{2}$ Department of Molecular Genetics, University of Toronto, Toronto, Ontario M5S 1A8, Canada Correspondence: Jeffrey L Wrana

E-mail: wrana@lunenfeld.ca

\section{References}

1 Farin HF, Jordens I, Mosa MH, et al. Nature 2016; 530:340-343.

2 Sato T, van Es JH, Snippert HJ, et al. Nature 2011; 469:415-418.

3 de Lau W, Peng WC, Gros P, et al. Genes Dev 2014; 28:305-316.

4 Bartscherer K, Boutros M. EMBO Rep 2008; 9:977-982.

5 Willert K, Nusse R. Cold Spring Harb Perspect Biol 2012; 4:a007864.

6 Stanganello E, Scholpp S. J Cell Sci 2016; 129:665-672.

7 Steinhauer J, Treisman JE. Curr Opin Genet Dev 2009; 19:308-314.

8 Zhang L, Wrana JL. Curr Opin Genet Dev 2014; 27:14-19.

9 de Groot RE, Farin HF, Macůrková M, et al. PLoS One 2013; 8: 76971.

10 Kabiri Z, Greicius G, Madan B, et al. Development 2014; 141:2206-2215. 